\title{
On the equation of degree 6
}

\author{
C. De Concini*, C. Procesi* and M. Salvetti*
}

\begin{abstract}
In this paper we study the Schwarz genus for the covering of the space of polynomials with distinct roots by its roots.

We show that, for the first unknown case (degree 6), the genus is strictly less than the one predicted by dimension arguments, contrary to what happens in all other reflection groups.
\end{abstract}

Mathematics Subject Classification (2000). 55R37.

Keywords. Schwarz genus, symmetric group, braid group, group homology.

\section{Introduction}

If one wants to find the roots of an equation $x^{m}+a_{1} x^{m-1}+\cdots=0$ of degree $m$ over $\mathbb{C}$ there are two immediate reductions. The first is to change variables and set $a_{1}=0$, the second is to reduce to the case of distinct roots or to a lower degree equation.

This leads one to consider the roots as unramified covering of the open set $\mathcal{P}_{m}$ of $\mathbb{C}^{m-1}$, the complement of the discriminant hypersurface.

At the end the problem is, to give a minimum number of monodromous functions of the coefficients (which necessarily are defined only in some open subsets of $\mathcal{P}_{m}$ ) with values the roots. It is a simple topological fact which we will recall presently, that one can always exhibit $m$ such functions (covering the entire $\mathcal{P}_{m}$ ). It was known that, if $m=p^{k}$ is a prime power this is always the minimum possible ([Va]).

In general some weak lower bounds are known.

In this paper we will study the first non prime power case $m=6$ and prove that for an equation of degree 6 one can express the distinct roots through 5 functions of the coefficients (and 5 is the minimum).

This is formalized through the notion of The Schwarz genus of a fibration (Definition 1.1).

*Partially supported by M.U.R.S.T. $40 \%$. 
Let $\mathcal{P}_{n+1}$ be the space of monic polynomials of degree $n+1$ over $\mathbb{C}$ with distinct roots, the complement of the discriminant hypersurface and let

$$
\Delta:=\left\{\left(z_{1}, z_{2}, \ldots, z_{n+1}\right\} \in \mathbb{C}^{n+1} \mid z_{i} \neq z_{j}, \forall i \neq j\right\}, \quad \mathcal{P}_{n+1}=\left(\mathbb{C}^{n+1}-\Delta\right) / S_{n+1}
$$

( $\Delta$ is the big diagonal).

Problem. Compute the Schwarz genus $g(n+1):=g\left(\gamma_{n+1}\right)$ for the covering

$$
\gamma_{n+1}: \mathbb{C}^{n+1}-\Delta \rightarrow \mathcal{P}_{n+1}=\mathbb{C}^{n+1}-\Delta / S_{n+1}
$$

One has:

Theorem 7.4. The genus $g(6)=5$ so 5 holomorphic functions suffice to compute the roots of a polynomial of degree 6 .

The methods in principle could be extended but at the moment we could overcome the computational difficulties only with the help of a computer program which seems too complex to perform in the next unknown case $m=10$.

\section{The Schwarz genus}

Definition 1.1 [Sc]. For a Galois covering $\gamma: A \rightarrow B$ the Schwarz genus $g(\gamma)$ is the minimum number for which one can cover the base with open sets $U_{i}$ so that the covering, restricted to $U_{i}$ is trivial.

Remark. The notion of genus is a generalization, to fibrations, of the LuisternikSchnirlemann category of a space.

This number is interpreted by Smale in the case of $\gamma_{n+1}$, as a measure of topological complexity for any algorithm that should compute the roots of a polynomial (cf. $[\mathrm{Sm}])$.

As already remarked we can normalize to the case in which the sum of the roots is 0 , this is then the simplest example of reflection arrangement, and the question may be asked for all such arrangements. In [DS1] it has been shown that, for all reflection arrangements except type $\mathcal{A}_{n}$ the genus of the associated covering is $n+1$ where $n$ is the complex dimension of the arrangement and the cohomological dimension of the corresponding Artin braid group.

For type $\mathcal{A}_{n}$ the same statement is true when $n+1=p^{k}$ is a prime power (cf. $[\mathrm{Va}])$.

In this paper we show that the previous statement is not always true. In fact we will verify that, for the first case $n+1=6$ of non prime powers, $g(6)=5$, 
(see Theorem 7.4), while the estimate given by the cohomological dimension is $g(6) \leq 6$.

Let us recall briefly the main ideas of Schwarz for the computation of this genus. We will restrict our discussion to finite Galois coverings, although Schwarz treats a much more general case. The proposition that follows is extracted from ([Sc]) and it is included for convenience of the reader.

Let then $\gamma: A \rightarrow B=A / W$ be a finite covering associated to the free action of a finite group $W$ on a space $A$. To this covering and any positive integer $k$ one can apply the join construction, set:

$$
A_{\gamma}^{k *}:=A *_{B} A * \cdots *_{B} A:=\left\{\sum_{i=1}^{k} t_{i} a_{i} \mid 0 \leq t_{i}, \sum_{i} t_{i}=1, \gamma\left(a_{i}\right)=\gamma\left(a_{j}\right), \forall i, j\right\} .
$$

In particular $A_{\gamma}^{k *}$ is a subset of the $k$-fold join. We have a fibration $\gamma_{k}: A_{\gamma}^{k *} \rightarrow B$, with fibers the join $F^{k *}$ of the fibers of $\gamma$. Then we have (assume $B$ paracompact):

Proposition 1.2. The genus $g(\gamma) \leq k$ if and only if the fibration $\gamma_{k}: A_{\gamma}^{k *} \rightarrow B$ has a section.

Proof. Let $V_{i}$ be the open subset of $A_{\gamma}^{k *}$ where the $i^{t h}$ coordinate $t_{i} \neq 0$, in $V_{i}$ we have a copy of $A$ as the points where the $i^{t h}$ coordinate $t_{i}=1$. By the standard properties of the join the space $A$ is a deformation retract of $V_{i}$. If $s: B \rightarrow A_{\gamma}^{k *}$ is a section of $\gamma_{k}$ define $U_{i}:=s^{-1}\left(V_{i}\right)$, the $U_{i}$ cover $B$ and by the previous remarks the fibration restricted to $U_{i}$ has a section, since it is a Galois fibration it is then trivial.

Conversely if $\gamma$ restricted to $U_{i}$ has a section $s_{i}$ for some covering $U_{1}, \ldots, U_{k}$ let $f_{i}$ be a partition of unity relative to this covering, we have that the map $\sum_{i=1}^{k} f_{i}(b) s_{i}(b)$ is a well defined section of $A_{\gamma}^{k *}$.

It is useful to think of the join construction as a base change. Given $k$, consider the $k$-fold join $W^{* k}$ of the group $W$.

We act on the right with $W$ on $W^{* k}$ by $\left(\sum_{i} t_{i} w_{i}, w\right) \rightarrow \sum_{i} t_{i} w_{i} w$ and have

$$
A_{\gamma}^{k *}=W^{* k} \times_{W} A, \quad \rho_{k}: A_{\gamma}^{k *} \rightarrow W^{* k} / W .
$$

The fibration $W \rightarrow W^{* k} \rightarrow W^{* k} / W$ gives $W^{* k} / W$ as the $k^{t h}$ step of Milnor's construction of a classifying space of $W$ (cf. [Mi]). $W^{* k} / W$ is itself a classifying space for $W$ fibrations over $\mathrm{CW}$-complexes of dimension $<k$ (if one wishes one can take $W^{* k} / W$ as the $k-1$ skeleton of a classifying space).

It may be useful to think of the right action of $W$ on $W^{* k}$ as a left action by $w\left(\sum_{i} t_{i} w_{i}\right):=\sum_{i} t_{i} w_{i} w^{-1}$, then $W^{* k} \times_{W} A$ is the space of $W$ orbits of the diagonal action. 
Given a space $X$ over which $W$ acts on the left consider the quotient $(X \times A) / W$. $(X \times A) / W$ fibers over $B$, by $\gamma_{X}(x, a)=\gamma(a)$ and over $X / W$ by $\rho(x, a)=[x]$, where $[x]:=\bar{\gamma}(x)$ denotes the orbit of $x$ in $X / W$. We claim that:

Proposition 1.3. i) There is a 1-1 correspondence between sections of $\gamma_{X}$ : $(X \times A) / W \rightarrow B$ and $W$ equivariant maps $\phi: A \rightarrow X$.

ii) If $s: B \rightarrow(X \times A) / W$ is a section of $\gamma_{X}$ and $\phi: A \rightarrow X$ the corresponding equivariant map we have a commutative diagram:

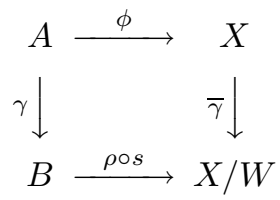

and the covering $\gamma: A \rightarrow B$ is the pullback, under $\rho \circ$ s of the covering $\bar{\gamma}: X \rightarrow X / W$.

Proof. i) Given a pair $(x, a) \in X \times A$ let us denote by $[x, a]$ its class in the $W$-orbit space. Given an equivariant map $\phi: A \rightarrow X$, consider the map $\sigma$ : $A \rightarrow X \times A \rightarrow(X \times A) / W$ given by $\sigma(a)=[\phi(a), a]$. We have that for $w \in$ $W, \sigma(w(a))=[\phi(w a), w a]=[w \phi(a), w a]=[\phi(a), a]=\sigma(a)$ therefore $\sigma$ factors through $B=A / W$ to a section of $\gamma_{X}$. Conversely given a section $s$ and $a \in A$ we can choose for $s(\gamma(a)) \in(X \times A) / W$ as representative in $X \times A$ a unique element $(\phi(a), a)$ and this defines the equivariant map.

ii) By definition $s(\gamma(a))=\sigma(a)=[\phi(a), a]$ which composed with $\rho$ gives $[\phi(a)]=\bar{\gamma}(\phi(a))$, or $\rho \circ s \circ \gamma=\bar{\gamma} \circ \phi$ as desired.

In the case of $A_{\gamma}^{k *}=\left(W^{* k} \times A\right) / W$ the previous proposition gives

Corollary 1.4. $\rho_{k} \circ s_{k}$ is a classifying map for the covering $\gamma: A \rightarrow B$.

The homology $H_{k-1}\left(W^{* k}, \mathbb{Z}\right)$ can be computed using the chain complex associated to the canonical cell decomposition of $W^{* k}$ (given by the join construction), as the group of $k-1$-cycles. Notice that this chain complex is a free resolution of the trivial $W$-module $\mathbb{Z}$ up to degree $k-1$.

This implies that, given any projective resolution $\left(C_{*}, \partial\right)$ of the trivial $W$ module $\mathbb{Z}$, there are two projective $W$-modules $P_{1}, P_{2}$, such that:

$$
H_{k-1}\left(W^{* k}, \mathbb{Z}\right) \oplus P_{1}=\partial C_{k} \oplus P_{2}
$$

In particular $H^{*}\left(W, H_{k-1}\left(W^{* k}\right)\right)=H^{*}\left(W, \partial C_{k}\right)$. 


\section{The universal obstruction}

Proposition 1.2 allows us to use obstruction theory to help to compute $g(\gamma)$ when $B$ is a finite CW-complex (cf. [St]). One uses the fact that $\pi_{i}\left(W^{* k}\right)=0, \forall i<k-1$, to deduce that $g(\gamma) \leq \operatorname{dim} B+1$. Let $\operatorname{dim} B=n$. In order to decide whether $g(\gamma) \leq n$ one has to compute the following obstruction class.

Consider $H^{*}\left(W, H_{n-1}\left(W^{* k}\right)\right)=H^{*}\left(W, \partial C_{n}\right)$. Recall that this can be computed as the cohomology of the cochain complex $\operatorname{Hom}\left(C_{*}, \partial C_{n}\right)$.

In particular, the map $\partial_{n}: C_{n} \rightarrow \partial C_{n}$ is clearly a cocycle $\left[\partial_{n}\right] \in$ $H^{n}\left(W, H_{n-1}\left(W^{* k}\right)\right)$ (we remark that this class does not depend on the choice of the resolution $\left(C_{*}, \partial\right)$ ). We can then consider the local system $\mathcal{H}_{n-1}\left(W^{* n}\right)$ on $B$, and we get the cocycle $c=\rho^{*}\left[\partial_{n}\right]$, where $\rho: B \rightarrow B W$ is a classifying map for our covering and a cohomology class $[c] \in H^{n}\left(B, \mathcal{H}_{n-1}\left(W^{* n}\right)\right)$. One has (see [St] Theorem 34.2, Lemma 32.7):

Theorem 2.1. $\partial_{n}$ and $c$ are obstruction cocycles for the respective problems of extending a section to the $n$-dimensional skeleton.

If $B$ is $n$-dimensional then $g(\gamma) \leq n$ if and only if $[c]=0$.

When $B=\mathcal{B}_{G}$ is the classifying space of a group $G$ (as in our case in which $\mathcal{P}_{n+1}$ is the classifying space of the Braid group $B_{n+1}$ ), we have that the Galois covering is given by a homomorphism $\rho_{*}: G \rightarrow W$ (induced by the homotopy exact sequence of the fibration $\gamma$ ) and one can think of the cohomology $H^{n}\left(B_{G}, \mathcal{H}_{n-1}\left(W^{* n}\right)\right)=H^{n}\left(G, H_{n-1}\left(W^{* n}\right)\right)$ as the cohomology of the group $G$ with coefficients in the module $H_{n-1}\left(W^{* n}\right)$ where the action is induced, via the homomorphism, by the $W$ action.

If we now denote by $\left(E_{*}, d\right)$ a projective resolution of the trivial $G$-module $\mathbb{Z}$, then the homomorphism $\rho_{*}$ induces a map of chain complexes $\rho_{*}: E_{*} \rightarrow C_{*}$, which induces the map of complexes

$$
\rho^{*}: \operatorname{Hom}_{W}\left(C_{*}, H_{n-1}\left(W^{* n}\right)\right) \rightarrow \operatorname{Hom}_{W}\left(E_{*}, H_{n-1}\left(W^{* n}\right)\right)
$$

and the map $\rho^{*}: H^{*}(W, M) \rightarrow H^{*}(G, M)$, in cohomology.

We may summarize the various ingredients introduced:

Theorem 2.2. Let $G$ and $W$ be two groups, with $W$ finite. Let $A \rightarrow B$ be a Galois covering with fiber $W$ and denote by $\rho_{*}: G \rightarrow W$ the corresponding homomorphism. Assume $\operatorname{dim} B \leq n$. The following statements are equivalent:

1) The genus of the covering $A \rightarrow B$ is less than or equal to $n$.

2) The fibration $W^{* k} \times_{W} A \rightarrow B$ has a section.

3) The classifying map of the covering $\gamma: A \rightarrow B$ factors through the $n-1$ dimensional space $W^{* n} / W$.

4) For every $W$ module $M$ the map in cohomology $\rho^{*}: H^{n}(W, M) \rightarrow H^{n}(G, M)$ is 0 .

5) The cohomology class of $\rho^{*}\left[\partial_{n}\right]$ is 0 . 
Proof. By Proposition 1.2, 1) and 2) are equivalent. 2) implies 3) by Corollary 1.4. 3) clearly implies 4) and also 4) clearly implies 5). Finally 5) implies 2) by Theorem 2.1.

\section{Braid and symmetric groups}

We apply now the previous theory to $G=B_{n+1}, W=S_{n+1}$ and to the canonical quotient homomorphism $B_{n+1} \rightarrow S_{n+1}$, so that the fibration we get is, up to homotopy, the fibration $\gamma_{n+1}: \mathbb{C}^{n+1}-\Delta \rightarrow \mathcal{P}_{n+1}$, considered in the introduction.

In this case $\mathcal{P}_{n+1}$ can be replaced by a finite CW-complex of dimension $n$ and we will use the very explicit constructions given in ([Sa], [DS1]). To say that $[c]=0$ where $c=\rho^{*}\left[\partial_{n}\right]$ means that the cocycle $\partial_{n}$ restricted to $E_{n}$ is a coboundary, or in other words that there exists a map $g: E_{n-1} \rightarrow \partial C_{n}$ such that the diagram:

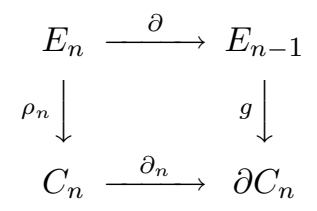

commutes.

All the maps are linear with respect to the group algebra $S$ of the braid group, so it is convenient to reformulate this statement as follows. Let $R$ be the integral group algebra of the symmetric group and define $D_{*}:=E_{*} \otimes_{S} R$, then $D_{*}$ is a subcomplex of $C_{*}$ and denoting by $i$ its inclusion the diagram becomes by abuse of notations:

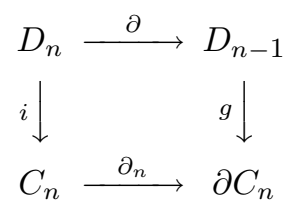

where now all the modules are free $R$ modules and the maps $R$-linear.

Recall from [DS1] that $D_{n}=R e, D_{n-1}=\oplus_{k=1}^{n} R e_{k}, \partial e=\sum_{k} A_{k} e_{k}$ with the $A_{k}$ 's defined as follows. For each $1 \leq k \leq n$ set

$$
\begin{gathered}
\mathcal{A}_{k}:=\left\{\sigma:=(\sigma(1), \sigma(2), \ldots, \sigma(n+1)) \in S_{n+1} \mid\right. \\
\sigma(1)<\sigma(2) \cdots<\sigma(k-1)<\sigma(k) ; \quad \sigma(k+1)<\sigma(k+2) \cdots<\sigma(n)<\sigma(n+1)\} .
\end{gathered}
$$

Then

$$
A_{k}=\sum_{\sigma \in \mathcal{A}_{k}} \epsilon(\sigma) \sigma,
$$

$\epsilon(\sigma)$ is the sign. 
Therefore:

Lemma 3.1. The map $g$ exists if and only if there exist elements $x_{k} \in \partial C_{n}$ with $\sum_{k} A_{k} x_{k}=\sum_{k} A_{k} e_{k}$.

Proof. A map $g: D_{n-1} \rightarrow \partial C_{n}$ is completely determined by the values $x_{k}=g\left(e_{k}\right)$ and, since $D_{n}$ is generated by $e$ the equality $g \circ \partial=a \circ i$ is equivalent to $g \circ \partial(e)=$ $\partial_{n} \circ i(e)$ or $\sum_{k} A_{k} x_{k}=\sum_{k} A_{k} e_{k}$.

We can further reformulate the last statement as follows.

By definition, given $x_{k} \in \partial C_{n}$ there exists $y_{k} \in C_{n}$ such that $x_{k}=\partial y_{k}$ so that the map $g$ exists if and only if there exist elements $y_{k} \in C_{n}$ with $\sum_{k} A_{k} \partial y_{k}=$ $\sum_{k} A_{k} e_{k}=\partial e$, or $\partial\left(e-\sum_{k} A_{k} y_{k}\right)=0$. Again by exactness of $C_{*}$ this means that there exists an element $b \in C_{n+1}$ with $\partial(b)=e-\sum_{k} A_{k} y_{k}$.

Finally this can be reinterpreted as follows. Let $I=\sum_{k} A_{k} R$ be the right ideal in $R$ generated by the elements $A_{k}$. On the category of $R$ modules define the functor

$$
N \rightarrow T(N):=N / \sum_{k} A_{k} N=R / I \otimes_{R} N .
$$

Take the complex $T\left(C_{*}\right)=R / I \otimes_{R} C_{*}$ whose homology is $H_{*}\left(S_{n+1}, R / I\right)$.

By construction, the element $\bar{e} \in T\left(D_{n}\right) \subset T\left(C_{n}\right)$, class of $e$, is a cycle and so it gives a homology class $[\bar{e}] \in H_{n}\left(T\left(D_{*}\right)\right)$. Denoting by $j$ the inclusion of $T\left(D_{n}\right)$ into $T\left(C_{n}\right)$, take $j[\bar{e}] \in H_{n}\left(S_{n+1}, R / I\right)$. We can thus interpret the previous discussion as:

Theorem 3.2. $\rho^{*}\left[\partial_{n}\right]$ is a coboundary if and only if $j[\bar{e}]=0$.

Notice now that the exact sequence $0 \rightarrow D_{*} \rightarrow C_{*} \rightarrow C_{*} / D_{*} \rightarrow 0$ of complexes of free $R$ modules induces an exact sequence $0 \rightarrow T\left(D_{*}\right) \rightarrow T\left(C_{*}\right) \rightarrow$ $T\left(C_{*} / D_{*}\right) \rightarrow 0$. From the long exact sequence of homology $H_{n+1}\left(T\left(C_{*} / D_{*}\right)\right) \stackrel{\partial}{\longrightarrow}$ $H_{n}\left(T\left(D_{*}\right)\right) \stackrel{j}{\rightarrow} H_{n}\left(T\left(C_{*}\right)\right) \ldots$ it follows that $j[\bar{e}]=0$ if and only if $[\bar{e}]=\partial(u)$, $u \in H_{n+1}\left(T\left(C_{*} / D_{*}\right)\right)$.

Since $D_{n+1}=0$ we have that $H_{n}\left(T\left(D_{*}\right)\right)$ are the cycles of the map $T\left(D_{n}\right) \stackrel{d}{\longrightarrow} T\left(D_{n-1}\right)$ and most of the computational difficulty lies in computing $H_{n+1}\left(T\left(C_{*} / D_{*}\right)\right)$. In the next sections we will start to describe in more detail the module $R / I$ and make a few simplifications which will allow us at the end to reduce the computational complexity, in case $n=5$ to a manageable size.

\section{The module $R / I$}

In order to continue we must deduce some properties of $R / I$ and to describe algorithmically the complex $T\left(C_{*} / D_{*}\right)$. 
Theorem 4.1. We have a direct sum decomposition, as $S_{n}$ modules, $R=\mathbb{Z}\left[S_{n+1}\right]$ $=I \oplus \mathbb{Z}\left[S_{n}\right]$.

Proof. First of all let us make the following remarks. By our description of the complex $D_{*}$, we can identify $H_{n}\left(D_{*}\right)=\left\{r \in R \mid r A_{k}=0, \forall k\right\}$. Also from its very definition, the complex $D_{*}$ computes the homology of the total space $A:=$ $\mathbb{C}^{n+1}-\Delta$, of our fibration and we get $[\mathrm{O}-\mathrm{S}]$ that $H_{n}\left(D_{*}\right)$ is a free $\mathbb{Z}$ module of rank $\mathrm{n}$ !.

Let us now use the (integral) trace form $t(a):=\frac{1}{n !} \operatorname{Tr}\left(a_{L}\right)$ associated to the regular representation, $a_{L}: \mathbb{Z}\left[S_{n+1}\right] \rightarrow \mathbb{Z}\left[S_{n+1}\right], a_{L}(u)^{n !}=a u$. This is non degenerate over $\mathbb{Z}$.

Under this form given $r \in R$ :

$$
r \in H_{n}\left(D_{*}\right) \Longleftrightarrow 0=t\left(x r A_{k}\right)=t\left(r A_{k} x\right) \forall k, \forall x \in S_{n+1}
$$

thus $H_{n}\left(D_{*}\right)$ is the orthogonal complement with respect to the form $t(a b)$, to the right ideal $I=\sum_{k=1}^{n} A_{k} \mathbb{Z}\left[S_{n+1}\right]$.

Since $\operatorname{rk} H_{n}\left(D_{*}\right)=n !, \operatorname{rk} R / I=n$ !. Therefore in order to prove our claim, it suffices to show that $\mathbb{Z}\left[S_{n+1}\right]=I+\mathbb{Z}\left[S_{n}\right]$. Given a permutation $\sigma \in S_{n+1}$, consider $k:=n+1-\sigma(n+1)$. If $k=0$, we have nothing to show since $\sigma \in S_{n}$. Otherwise we proceed by induction on $k$.

Given a permutation $\tau=\left(i_{1}, i_{2}, \ldots, i_{n+1}\right) \in \mathcal{A}_{k}$ we clearly have that $i_{n+1} \geq$ $n+1-k$. Furthermore, if $i_{n+1}=n+1-k$, we must necessarily have that

$$
\tau=\tau_{k}:=(n+1-(k-1), \ldots, n+1,1,2,3, \ldots, n-k, n+1-k) .
$$

Thus, we can write $\tau=(-1)^{k(n+1-k)} A_{k}+b$, with $b$ a linear combination of permutations $\gamma$ for which $n+1-\gamma(n+1)<k$. Our inductive hypothesis then implies that $\tau \in I+\mathbb{Z}\left[S_{n}\right]$.

Take now an arbitrary permutation $\sigma$ with $\sigma(n+1)=n+1-k$. We can write $\sigma=\tau_{k} \gamma, \gamma \in S_{n}$. Since $I+\mathbb{Z}\left[S_{n}\right]$, is clearly stable under right multiplication by elements in $S_{n}$, everything follows.

Remark. We have that, $R / I$ as a $\mathbb{Z}\left[S_{n}\right]$-module is free of rank 1 .

Thus $H_{i}\left(S_{n}, R / I\right)=0, \forall i>0$. Since $n+1=\left[S_{n+1}: S_{n}\right]$, multiplication by $n+1$ on $H_{i}\left(S_{n+1}, R / I\right)$ can be written as the composition of the restriction and corestriction

$$
n+1: H_{i}\left(S_{n+1}, R / I\right) \stackrel{\text { res }}{\longrightarrow} H_{i}\left(S_{n}, R / I\right) \stackrel{\text { cores }}{\longrightarrow} H_{i}\left(S_{n+1}, R / I\right),
$$

we deduce that $(n+1) H_{i}\left(S_{n+1}, R / I\right)=0, \forall i>0$ (cf. [B], Prop. 9.5).

The previous discussion gives an explicit algorithm to perform the projection:

$$
\mathbb{Z}\left[S_{n+1}\right]=\mathbb{Z}\left[S_{n}\right] \oplus I \stackrel{\gamma}{\rightarrow} \mathbb{Z}\left[S_{n}\right]
$$

With this explicit map the computation of the complex $R / I \otimes_{R} C_{*}$ can be developed. 


\section{The complex}

We now recall the free resolution $C_{*}$ of $\mathbb{Z}$ by $R$ modules described in [DS1]. Denote by $\mathcal{N}$ the set of $n$ nodes of $A_{n}$ which we identify with the set of simple transpositions $(h, h+1)$ and we order linearly. Let $\mathcal{B}_{k}$ denote the set of flags of $k$ subsets of $\mathcal{N}$ with a total of $k$ elements, i.e. sequences $b:=\mathcal{N} \supseteq \Gamma_{1} \supseteq \Gamma_{2} \supseteq \cdots \supseteq \Gamma_{k}$, such that $\sum\left|\Gamma_{i}\right|=k$.

Then $C_{k}$ is a direct sum $\oplus_{b \in \mathcal{B}_{k}} \mathbb{Z}\left[S_{n+1}\right] e_{b}$ and the differential $\partial e_{b}$ is explicitly described as follows. Let $S_{\Gamma_{i}}$ the parabolic subgroup generated by $\Gamma_{i}$ and $S_{\Gamma_{i}}^{\Gamma_{i} \backslash\{\tau\}}$ the coset representatives of shortest length of $\Gamma_{i} \backslash\{\tau\}$ in $\Gamma_{i}$. Define a $\mathbb{Z}\left[S_{n+1}\right]_{\text {- }}$ linear differential $\partial_{k}: C_{k} \rightarrow C_{k-1}$ by

$$
\partial_{k} e_{b}=\sum_{\substack{1 \leq i \leq k \\\left|\Gamma_{i}\right|>\left|\Gamma_{i+1}\right|}} \sum_{\substack{\tau \in \Gamma_{i} \\ \beta^{-1} \Gamma_{i+1} \beta \subset S_{\Gamma_{i} \backslash \Gamma_{i} \backslash\{\tau\}}}}(-1)^{\alpha(\Gamma, i, \tau, \beta)} \beta e_{b^{\prime}}
$$

where $b^{\prime}=\left(\Gamma_{1}, \ldots, \Gamma_{i-1}, \Gamma_{i} \backslash\{\tau\}, \beta^{-1} \Gamma_{i+1} \beta, \ldots, \beta^{-1} \Gamma_{k} \beta\right) \quad$ and

$$
\alpha(\Gamma, i, \tau, \beta)=i \ell(\beta)+\sum_{j=1}^{i-1}\left|\Gamma_{j}\right|+\mu\left(\Gamma_{i}, \tau\right)+\sum_{j=i+1}^{d} \sigma\left(\beta, \Gamma_{j}\right) .
$$

Here $\ell$ is the standard length function, $\sigma\left(\beta, \Gamma_{j}\right)$ is the number of inversions in the map $\Gamma_{j} \rightarrow \beta^{-1} \Gamma_{j} \beta \subset \Gamma_{i} \backslash \tau$ and $\mu\left(\Gamma_{i}, \tau\right)$ is the number of reflections in $\Gamma_{i}$ which are less than or equal to $\tau$ in the linear ordering.

Using the decomposition and projection $(P r)$ we identify $R / I \otimes_{R} C_{k}$ with $\oplus_{b \in \mathcal{B}_{k}} \mathbb{Z}\left[S_{n}\right] e_{b}$. As for the boundary, $\mathbb{Z}\left[S_{n+1}\right]=\mathbb{Z}\left[S_{n}\right] \oplus I \stackrel{\gamma}{\rightarrow} \mathbb{Z}\left[S_{n}\right]$ induces termwise a map

$$
\oplus_{b \in \mathcal{B}_{k}} \mathbb{Z}\left[S_{n+1}\right] e_{b}=\oplus_{b \in \mathcal{B}_{k}}\left(\mathbb{Z}\left[S_{n}\right] \oplus I\right) e_{b} \stackrel{\gamma}{\rightarrow} \oplus_{b \in \mathcal{B}_{k}} \mathbb{Z}\left[S_{n}\right] e_{b} .
$$

So the boundary in $R / I \otimes_{R} C_{*}$ is the composition:

$$
\Delta_{k}: \oplus_{b \in \mathcal{B}_{k}} \mathbb{Z}\left[S_{n}\right] e_{b} \stackrel{\partial}{\rightarrow} \oplus_{c \in \mathcal{B}_{k-1}}\left(\mathbb{Z}\left[S_{n}\right] \oplus I\right) e_{c} \stackrel{\gamma}{\rightarrow} \oplus_{c \in \mathcal{B}_{k-1}} \mathbb{Z}\left[S_{n}\right] e_{c} .
$$

Further simplification comes from the following remark.

Proposition 5.1. Let $\mathcal{B}_{k}^{h} \subset \mathcal{B}_{k}$ denote the set of flags of subsets of $\{1, \ldots, \hat{h}, \ldots, n\}$ with cardinality $k$. Then the two subcomplexes $C_{*}(1), C_{*}(n)$ of $R / I \otimes_{R} C_{*}$ which are generated respectively by

$$
\oplus_{b \in \mathcal{B}_{k}^{1}} R / I e_{b}, \quad \oplus_{b \in \mathcal{B}_{k}^{n}} R / I e_{b}
$$

are acyclic. 
The same statement is true in degree $\geq 2$ for the subcomplex given by their sum.

Proof. From formulas of [DS1] the subcomplex $C_{*}(n)$ which is generated by

$$
\oplus_{b \in \mathcal{B}_{k}^{n}} \mathbb{Z}\left[S_{n}\right] e_{b}
$$

equals the acyclic complex $C_{*}^{n-1}$ of $S_{n}$. Clearly the theory is symmetric, so the same is true for the other subcomplex.

Now we see that the intersection $C_{*}(1) \cap C_{*}(n)$ is a subcomplex of free $\mathbb{Z}\left[S_{n}\right]$ modules with bases corresponding to flags with no nodes $1, n$. Again from formulas in [DS1] it is clear that this subcomplex splits (in degree $>0$ ) as a sum of $n$ copies of the acyclic complex $C_{*}^{n-2}$ of the group $S_{n-1}$. Therefore by looking at the Mayer-Vietoris sequence one concludes.

So we reduce to make computations on the quotient

$$
\tilde{C}_{*}:=R / I \otimes_{R} C_{*} /\left(C_{*}(1)+C_{*}(n)\right)
$$

which is a complex of free $\mathbb{Z}\left[S_{n}\right]$-modules with bases $e_{b}$, where the flag $b$ contains both 1 and $n$. Let $\tilde{\partial}$ be the boundary induced by $\bar{\partial}$.

Theorem 3.2 translates here to

Theorem 5.2. Let $b_{0} \in \mathcal{B}_{n}$ be the flag

$$
b_{0}:=(\{1, \ldots, n\} \supset \emptyset \supset \ldots) .
$$

The class $\left[\partial_{n}\right]$ is 0 iff $e_{b_{0}}$ belongs to the image of $\tilde{\partial}_{n+1}$.

\section{Computations}

Recall from Section 3 that the vanishing of the obstruction class $\left[\partial_{n}\right]$ is equivalent to that of a certain homology class $j(\bar{e}) \in H_{n}\left(S_{n+1}, R / I\right)$.

We want to compute $H_{i}\left(S_{6}, R / I\right)$ and show that $H_{5}\left(S_{6}, R / I\right)=0$.

As above, identify $R / I$ with $\mathbb{Z}\left[S_{n}\right]$ as $\mathbb{Z}\left[S_{n}\right]$-modules.

The projection $\gamma: \mathbb{Z}\left[S_{n+1}\right] \rightarrow \mathbb{Z}\left[S_{n}\right]$ is given by a matrix

$$
P \in \mathcal{M}(n ! \times(n+1) ! ; \mathbb{Z})
$$

which, can be determined following the algorithm described in the proof of Theorem 4.1.

More precisely, let us give an ordering $\sigma_{1}, \sigma_{2}, \ldots$ to the $n+1$ ! permutations so that the last $n$ ! of them fix $n+1$. Then each element in $\mathbb{Z}\left[S_{n+1}\right]$ corresponds 
to an integral vector with $n+1$ ! entries. Construct the $(n+1) ! \times(n \cdot(n+1) !)$ integral matrix $A$ whose columns are the $A_{k} \sigma_{j}, k=1, \ldots, n, j=1, \ldots,(n+1)$ !. Decompose $A$ as

$$
A=\left[\begin{array}{l}
B \\
C
\end{array}\right]
$$

where $B$ is of order $(n \cdot n !) \times(n \cdot(n+1) !)$ while $C$ is of order $(n !) \times(n \cdot(n+1) !)$. Then Theorem 4.1 is equivalent to

Theorem 6.1. $B$ is right invertible over $\mathbb{Z}$, i.e. there is an integral matrix $H$ with $B H=I_{n n !}$.

There exists a unique projection $\gamma: \mathbb{Z}\left[S_{n+1}\right] \rightarrow \mathbb{Z}\left[S_{n}\right]$ given by the matrix

$$
P=\left[\begin{array}{ll}
-C H & I_{n !}
\end{array}\right]
$$

where we indicate by $I_{n !}$ the identity matrix of order $n$ !, with:

$$
\left[\begin{array}{ll}
-C H & I_{n !}
\end{array}\right]\left[\begin{array}{l}
B \\
C
\end{array}\right]=0 .
$$

Computationally, one can find $C H$ by (integral) Gauss reduction of $A^{T}$.

We calculate (once for all) the matrix $P$. We also have algorithms which compute the boundary $\partial e_{b}$ and, plugging into the formula $(C o)$ we compute the integral matrix $\Delta_{k}$.

\section{The case $n=5$}

We now consider the case $n=5$ and compute $g(6)=g\left(\gamma_{6}\right)$.

We know by the general dimension argument that $g(6) \leq 6$, on the other hand the covering $\gamma_{6}$ contains a subcovering homeomorphic to $\gamma_{5}$ (by considering the polynomials with one given fixed root), therefore from the results of Vassiliev $g(6) \geq 5$. In order to determine whether $g(6)=6$ or $g(6)=5$ we have to compute the obstruction class.

Lemma 7.1. For $n=5$ the ranks of the free $\mathbb{Z}\left[S_{6}\right]$-modules $C_{*}$, resp. the free $\mathbb{Z}\left[S_{5}\right]$-modules $\tilde{C}_{*}$, in dimensions $i: 0,1,2,3,4,5,6$ are:

$$
\begin{gathered}
r k\left(C_{i}\right)=1,5,15,35,70,126,210, \\
r k\left(\tilde{C}_{i}\right)=0,0,1,5,15,35,70 .
\end{gathered}
$$

Proof. We just enumerate all flags with given cardinality, and those which contain both 1 and 5 . 
Now what we do is the following:

1) By using the general algorithm which produces the boundaries in $C_{*}$, with coefficients in $\mathbb{Z}\left[S_{6}\right]$, we compute the $(35 \times 70)$-submatrix (with values in $\mathbb{Z}\left[S_{6}\right]$ ) of the boundary $\partial_{6}$ which corresponds to those flags containing both 1 and 5 .

2) We multiply each column (on the left) by $\sigma \in S_{5}$ and transform that 35vector over $\mathbb{Z}\left[S_{6}\right]$ into a $(35 \times 120)$-vector over $\mathbb{Z}$ by using the projection matrix $P$.

So we obtain a $(35 \times 120=4200) \times(70 \times 120=8400)$-matrix which represents the boundary $\tilde{\partial}_{6}$ as $\mathbb{Z}$-modules.

3) We find the (Smith) normal form of such a matrix.

By general results $([\mathrm{B}])$ all homology groups are finite in positive dimension, in our case by the Remark of section 4, the number 6 kills homology. From the normal form of $\tilde{\partial}_{6}$ one soon has $H_{5}\left(\tilde{C}_{*}\right)$ which equals $H_{5}\left(S_{6}, R / I\right)$ by Proposition 5.1. By repeating the previous steps in lower dimension we find:

Theorem 7.2. The homology $H_{i}\left(S_{6} ; R / I\right)$ is for $i=0, \ldots, 5$

$$
H_{i}\left(S_{6} ; R / I\right): \quad \mathbb{Z} / 3 \mathbb{Z}, 0,0,0, \mathbb{Z} / 3 \mathbb{Z}, 0 .
$$

Proof. The cases $H_{k}\left(S_{6} ; R / I\right)$ with $k \leq 2$ are computed directly. For the remaining ones we make computations using $\tilde{C}_{*}$ as said above, the computations have been made with a program written using the package AXIOM.

Corollary 7.3. For $n=5$ the induced map $\rho^{*}$ in cohomology vanishes.

We have thus the main theorem:

Theorem 7.4. The genus $g(6)=5$ so 5 holomorphic functions suffice to compute the roots of a polynomial of degree 6 .

\section{References}

[B] K. Brown, Cohomology of Groups, GTM 87, Springer-Verlag, New York, 1982.

[DS] C. De Concini and M. Salvetti, Cohomology of Artin groups, Math. Res. Letters 3 (1996), 293-297.

[DS1] C. De Concini and M. Salvetti, Cohomology of Artin groups and Coxeter groups, Math. Res. Lett. 7 (2000), 213-232.

[Mi] J. Milnor, Construction of universal bundles. I, II, Ann. of Math. (2) 63 (1956), 272-284, 430-436.

[OS] P. Orlik and L. Solomon, Combinatorics and topology of complements of hyperplanes, Invent. Math. 56 no. 2 (1980), 167-189.

[Sa] M. Salvetti, The homotopy type of Artin groups, Math. Res. Letters 1 (1994), 565-577.

[Sc] A. S. Schwarz, The genus of a fibered space (Russian), Trudy Moskov. Mat. Obs. 10 (1961), 217-272.

[Sm] S. Smale, On the topology of algorithms I, Journal of complexity 3 (1987), 81-89. 
[St] N. Steenrod, The Topology of Fibre Bundles, Princeton University Press, Princeton, N. J., 1951.

[Va] V. A. Vassiliev, Topology of complements to discriminants, Amer. Math. Soc., Providence, RI, 1992, revised 1994.

\section{Procesi}

Dipartimento di Matematica

Università di Roma "La Sapienza"

Roma

Italy

e-mail: claudio@mat.uniroma1.it

\section{De Concini}

Dipartimento di Matematica

Università di Roma "La Sapienza"

Roma

Italy

e-mail: deconcin@mat.uniroma1.it

M. Salvetti

Dipartimento di Matematica

Università di Pisa

Pisa

Italy

e-mail: salvetti@dm.unipi.it

(Received: February 4, 2003)

(10) To access this journal online:

(20) http://www.birkhauser.ch 